Review

\title{
Something about Electron Dimension
}

\author{
${ }^{1}$ Relly Victoria V. Petrescu, ${ }^{2}$ Raffaella Aversa, ${ }^{2}$ Antonio Apicella, \\ ${ }^{3}$ Shuhui Li, ${ }^{4}$ Guanying Chen, and ${ }^{1}$ Florian Ion T. Petrescu \\ ${ }^{I}$ IFToMM-ARoTMM, Bucharest Polytechnic University, 313 Splaiul Independentei, Bucharest, (CE) Romania \\ ${ }^{2}$ Advanced Material Lab, Department of Architecture and Industrial Design, Second University of Naples, (CE) Italy \\ ${ }^{3}$ University of Alabama, United States \\ ${ }^{4}$ Harbin Institute of Technology and SUNY Buffalo, China
}

Article history

Received: 27-10-2016

Revised: 03-11-2016

Accepted: 24-11-2016

Corresponding Author: Florian Ion T. Petrescu IFToMM-ARoTMM, Bucharest Polytechnic University, 313 Splaiul Independentei, Bucharest, (CE) Romania Vice President of IFToMMARoTMM Bucharest branch Email: petrescuflorian@yahoo.com

\begin{abstract}
In this study will be exposed the theoretical principles necessary for the determination of the exact size of an electron in motion, depending on its speed of travel. Equations, aimed to accurately determines the radius $\mathrm{R}$ of the electron in motion, relating the electron moving speed $\mathrm{v}$ and its rest mass $\mathrm{m}_{0}$ are discussed. Mechanical moment of inertia of a sphere around of one of its diameters shall be determined by the relationship relating the total kinetic energy of one electron in motion, as a sum of the two components (translational and rotational). Using the theory of Louis de Broglie, which shows the conservation of the pulse, the wavelength (particle associated) has been calculated. Wave frequency (associated with the electron in motion) has been determined and moving electron kinetic energy has been estimated by subtracting the total electron rest energy from total electron energy in movement.
\end{abstract}

Keywords: Electron Radius, Electron Speed, Rest Mass, Speed of Light, Planck's Constant, Electron Kinetic Energy, Lorentz Expression, Louis De Broglie Theory, Pulse Conservation, Wavelength Particle Associated, Wave Frequency

\section{Introduction}

As known, matter at the fundamental level consists of quarks and leptons. Quarks combine to form hadrons, mostly baryons and mesons via the strong force and are presumed to still well confined. Among the baryons are the proton (the positive electrical charge) and neutrons (with zero electric charge) that they combine to form atomic nuclei of all chemical elements of the periodic table. Normally, a cloud of electrons (negative electric charge and exactly opposite to that of the proton) surrounds these cores. The assembly formed by a core and a cloud, which comprises negative electrons and positive protons, is an atom. Atoms can be arranged together to form larger and more complex structures such as molecules.

Chemistry is the science that describes how nuclei and electrons are combined to form various elements and molecules.

In a more cosmological vision matter and antimatter are considered. Each sub-particle of an atom may be counterbalanced with an (anti-) antimatter pair (e.g., electron-positron). An antimatter particle differs from its partner by the fact that all its various "fillers" (electric charge, spin, color charge, etc.) are opposite. However, such particles have the same mass.

Although the fundamental laws of physics do not indicate a preference for matter over antimatter, cosmological observations indicate that the universe consists almost entirely of matter.

The material can be found in several states or phases. The four most known states are solid, liquid, gas and plasma. There are also other a little more exotic states such as liquid crystal, Bose-Einstein, super-fluid and supercritical fluid (Bose-Einstein, Wikipedia). When the material passes from one state to another, it performs a phase transition. This phenomenon (which is associated to changes in physical parameters: Pressure, temperature, volume, density, energy, etc.) is studied in thermodynamic via the phase diagrams.

When several particles combine to form atoms, the total mass (at rest) of the assembly is smaller than the sum of the masses of (at rest) because in fact a part of the weight of components is converted the binding energy necessary to ensure the cohesion of the cluster (this phenomenon is reported as "the mass defect"). By speculating this defect, nuclear fusion energy is extracted. 
The electron, one of the particles of the atom having an elementary charge of negative sign, is fundamental in chemistry, because it participates in almost all types of chemical reactions and is a key element of matter chemical bonding. In physics, on the other hand, the electron is involved in a multitude of phenomena related to radiation effects. Electron features, which manifest them self at the atomic level, explain the electrical conductivity, thermal conductivity, the Vavilov-Cherenkov, incandescence, electromagnetic induction, luminescence, magnetism, electromagnetic radiation, optical reflection and superconductivity that are macroscopic widely exploited in the industrial applications (Cherenkov, Wikipedia).

Moreover, electron, with its lowest mass compared to any other charged particles, is regularly used in the study of matter. The concept of an indivisible amount of electric charge was developed from 1838 by the British naturalist Richard Laming to explain the chemical properties of atoms (Laming, Wikipedia). The electron was described as the corpuscle envisaged by Joseph John Thomson and his team of British physicists in 1897, following their work on cathode rays, developed its atomic model (Thomson, Wikipedia).

Albert Einstein proposed in 1905 an explanation of the photoelectric effect-electrons emitted by matter under the influence of light-that was reinterpreted as base in the development of the quantum theory (Einstein, Wikipedia). In 1912, Niels Bohr explained the spectral lines postulating the quantification of the orbit of the electrons from the hydrogen atom that was another argument supporting that theory (Bohr, Wikipedia).

The experiments carried out by Ernest Rutherford and others in 1921 have firmly established the structure of the atom as a positively charged nucleus surrounded by electrons of lower mass (Rutherford, Wikipedia). In 1923, the experimental results Arthur Compton convince a majority of physicists of the validity of quantum theory (Compton, Wikipedia). In 1924, Wolfgang Pauli principle defines the Pauli exclusion, which means that electrons possess spin (Pauli, Wikipedia). The same year, Louis de Broglie first postulated and then checked later that electrons exhibit wave-particle duality (Louis de Broglie, Wikipedia). In 1928, Paul Dirac published his model of the electron that introduced the concept of positron and antimatter (Dirac, Wikipedia). Other studies of the electron properties have led to more complete theories of matter and radiation.

\section{Materials and Methods}

When it comes to the determination of the size of atomic subatomic particles, as a rule the static diameters (or more precisely, the diameters of the particle at rest) is used. They have been determined by a variety of methods more or less empirical and with very approximate values (Halliday and Robert, 1966). These dimensions (that, depending on the particle could be of the order of nano,
Pico or less) are very difficult to determine both experimental and theoretically, even today when we dispose of many sophisticated characterization tools (Atomic force microscopes, nanoindenter, etc). However, the real characterization problem arises when these particles are in dynamic motion. In this dynamic condition it is mandatory to determine the actual precise dimensions in motion, which is a fact that makes things to become even more complex than they already are.

Previous experiences by the Authors have achieved the theoretical identification, with very high accuracy, the dimensions of elementary particles in motion, as far as they do not reach the speed of light (Petrescu and Calautit, 2016a; Petrescu et al., 2016).

For photons, which move at the speed of light, it was presented a special method for the determination of their dimensions, depending on the frequency of the specific electromagnetic wave (Petrescu and Calautit, 2016b).

In the present work we will be presenting the theoretical principles necessary for the determination of the exact size of an electron in motion in relation with its specific travelling speed.

The relationship 1 can accurately determines the radius of the electron in motion $R$, depending on its speed $\mathrm{v}$ and its rest mass $\mathrm{m}_{0}$. The speed of light in a vacuum is expressed with $\mathrm{c}$ and Planck's constant with h (Planck, Wikipedia):

$R=\sqrt{\frac{10}{8}} \cdot \frac{h \cdot \sqrt{c^{2}-v^{2}} \cdot \sqrt{c^{2}-\frac{v^{2}}{2}-c \cdot \sqrt{c^{2}-v^{2}}}}{\pi \cdot m_{0} \cdot c^{2} \cdot v}$

Mechanical moment of inertia of a sphere around of one of its axes could be determined by using the relationship 2 (Fig. 1), (Petrescu, 2012):

$J=\frac{2}{5} m \cdot R^{2}$

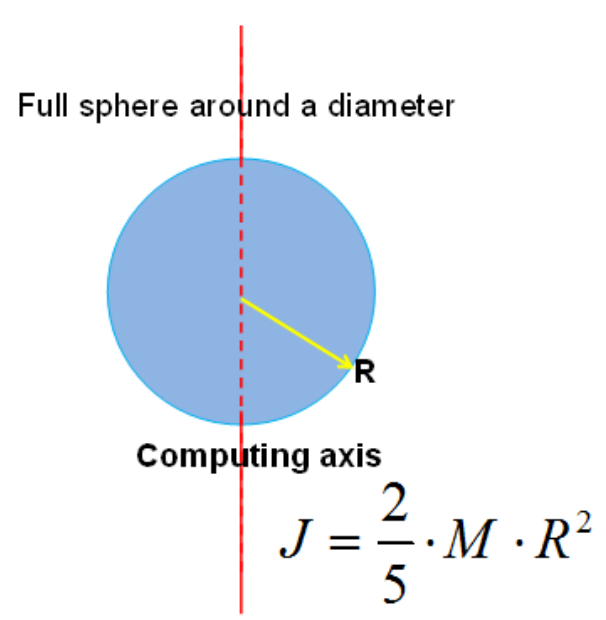

Fig. 1. Mass moment of inertia to a full sphere, determined around its symmetry axis 
The mass of an electron in motion can be determined then according to its speed and its rest mass using rehandled Lorentz expression 3 (Lorentz, Wikipedia):

$$
m=\frac{m_{0} \cdot c}{\sqrt{c^{2}-v^{2}}}
$$

The electron (as any elementary particle in motion) has two types of movements, one translational and another of rotation around its own axis. These two motions are corresponding to the two kinetic energies (energy due to translational motion of electron on its trajectory and the energy due to the rotating of the electron about a diameter axis); expression 4 shows the total kinetic energy of one electron in motion, as a sum of the two components (translational and rotational):

$$
E_{c}=\frac{1}{2} m \cdot v^{2}+\frac{1}{2} J \cdot \omega^{2}
$$

Using the two expressions 2 and 3, the relationship 4 takes the below forms 5 :

$$
\left\{\begin{array}{l}
E_{c}=\frac{1}{2} m \cdot v^{2}+\frac{1}{2} \cdot \frac{2}{5} \cdot m \cdot R^{2} \cdot \omega^{2} \\
E_{c}=\frac{1}{2} \frac{m_{0} \cdot c \cdot v^{2}}{\sqrt{c^{2}-v^{2}}}+\frac{1}{2} \cdot \frac{2}{5} \cdot \frac{m_{0} \cdot c \cdot R^{2}}{\sqrt{c^{2}-v^{2}}} \cdot \omega^{2}
\end{array}\right.
$$

One uses the expression 6 to write the particle pulse:

$$
p=m \cdot v=\frac{m_{0} \cdot c \cdot v}{\sqrt{c^{2}-v^{2}}}
$$

Using the theory of Louis de Broglie (de Broglie, Wikipedia), which describes the conservation of the pulse the wavelength particle associated may be calculated using relationship 7:

$$
\lambda=\frac{h}{p}=\frac{h \cdot \sqrt{c^{2}-v^{2}}}{m_{0} \cdot c \cdot v}
$$

Wave frequency (associated with the electron in motion) can be then determined using the relationship 8 (Halliday and Robert, 1966):

$\gamma=\frac{c}{\lambda}=\frac{c \cdot m_{0} \cdot c \cdot v}{h \cdot \sqrt{c^{2}-v^{2}}}=\frac{m_{0} \cdot c^{2} \cdot v}{h \cdot \sqrt{c^{2}-v^{2}}}$

The angular electron speed and its square are calculated using the expressions 9:

$$
\left\{\begin{array}{l}
\omega=2 \pi \gamma=\frac{2 \pi \cdot m_{0} \cdot c^{2} \cdot v}{h \cdot \sqrt{c^{2}-v^{2}}} \\
\omega^{2}=\frac{4 \pi^{2} \cdot m_{0}^{2} \cdot c^{4} \cdot v^{2}}{h^{2} \cdot\left(c^{2}-v^{2}\right)}
\end{array}\right.
$$

Considering expressions 9, expressions 5 may be written in the following expressions 10 :

$$
\left\{\begin{array}{l}
E_{c}=\frac{1}{2} \frac{m_{0} \cdot c \cdot v^{2}}{\sqrt{c^{2}-v^{2}}}+ \\
+\frac{1}{2} \cdot \frac{2}{5} \cdot \frac{m_{0} \cdot c \cdot R^{2}}{\sqrt{c^{2}-v^{2}}} \cdot \frac{4 \pi^{2} \cdot m_{0}^{2} \cdot c^{4} \cdot v^{2}}{h^{2} \cdot\left(c^{2}-v^{2}\right)} \\
E_{c}=\frac{1}{2} \frac{m_{0} \cdot c \cdot v^{2}}{\sqrt{c^{2}-v^{2}}} \cdot\left[1+\frac{8}{5} R^{2} \cdot \pi^{2} \frac{m_{0}^{2} \cdot c^{4}}{h^{2} \cdot\left(c^{2}-v^{2}\right)}\right]
\end{array}\right.
$$

Moving electron kinetic energy may be calculated through the expression 11 by subtracting the total electron rest energy from total electron energy in movement:

$$
\begin{aligned}
& E_{c}=E-E_{0}=m \cdot c^{2}-m_{0} \cdot c^{2}= \\
& =m_{0} \cdot c^{2} \cdot \frac{c-\sqrt{c^{2}-v^{2}}}{\sqrt{c^{2}-v^{2}}}
\end{aligned}
$$

By considering the two expressions 10 and 11 and combining them, the first term of relationships 12 is obtained, which represent the expression to evaluate the radius of an electron in movement. The second term from in Equation 12 is the initially mentioned relation 1:

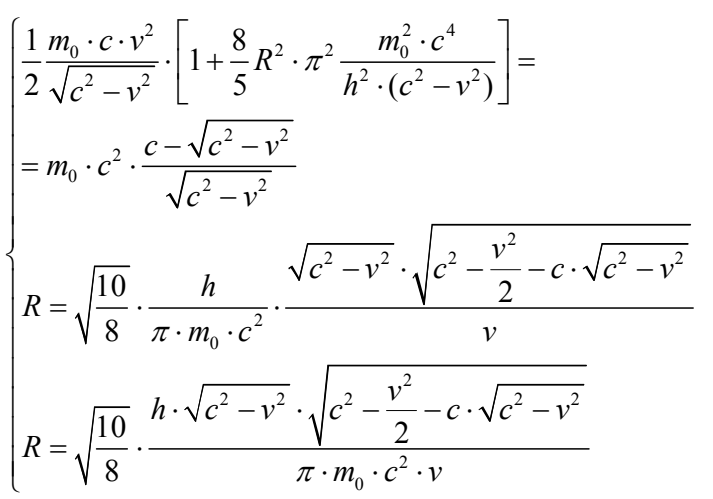

\section{Results and Discussion}

By using Equation 1 it may be now calculated the radius of any elementary particle in movement.

Table 1 shows the radius of an electron in movement (calculated with Equation 1) as a function of $\beta$ ( $\beta$ being the ratio between $v$ and $c$; expression 13):

$$
\beta=\frac{v}{c}
$$

By using the original method proposed by the authors may the radius of the electron in motion, may be determined with great precision according to the speed at which it moves. It can be seen from the results listed in Table 1 that the electron does not have a constant radius. The radius of the electron depends primarily on the speed of travel and, in the second row to its rest mass. 
Table 1. The electron radius in function of $\beta$

\begin{tabular}{llll}
\hline$\beta$ & 0.000009 & 0.00002 & 0.0001 \\
$R[\mathrm{~m}]$ & $4.93 \mathrm{E}-16$ & $4.07 \mathrm{E}-16$ & $8.15 \mathrm{E}-17$ \\
$\beta$ & 0.001 & 0.01 & 0.1 \\
$\mathrm{R}[\mathrm{m}]$ & $3.05 \mathrm{E}-16$ & $3.05 \mathrm{E}-15$ & $3.04 \mathrm{E}-14$ \\
$\beta$ & 0.2 & 0.3 & 0.4 \\
$\mathrm{R}[\mathrm{m}]$ & $6.04 \mathrm{E}-14$ & $8.94 \mathrm{E}-14$ & $1.16 \mathrm{E}-13$ \\
$\beta$ & 0.5 & 0.6 & 0.7 \\
$\mathrm{R}[\mathrm{m}]$ & $1.41 \mathrm{E}-13$ & $1.62 \mathrm{E}-13$ & $1.78 \mathrm{E}-13$ \\
$\beta$ & 0.8 & 0.9 & 0.99 \\
$\mathrm{R}[\mathrm{m}]$ & $1.83 \mathrm{E}-13$ & $1.66 \mathrm{E}-13$ & $7.47 \mathrm{E}-14$ \\
$\beta$ & 0.999 & 0.9999 & 0.99999 \\
$R[\mathrm{~m}]$ & $2.61 \mathrm{E}-14$ & $8.51 \mathrm{E}-15$ & $2.71 \mathrm{E}-15$ \\
$\beta$ & 0.999999 & 0.9999999 & 1 \\
$\mathrm{R}[\mathrm{m}]$ & $8.62 \mathrm{E}-16$ & $2.72 \mathrm{E}-16$ & $8.63 \mathrm{E}-17$ \\
\hline
\end{tabular}

From the presented table it may be determined the value of average radius of an electron 1.09756E-13 [m] and an electron maximum value $1.83152 \mathrm{E}-13$ [m] corresponding to a $\beta=0.8$.

The minimum electron radius value (to the real cases) is being about $8.15 \mathrm{E}-17$ [m] but it maybe to shrink more in cases of reaching the limiting conditions.

The electrons which move normally with the low speeds of approximately $0.01 \mathrm{c}$ will have the radius in value of $3.05 \mathrm{E}-15[\mathrm{~m}]$. Only this value can be found using the classic relations already known.

\section{Assumption Used}

The assumption that frequency of the particle associate wave is the same with rotation frequency of the particle (the rotation of the particle produces the associate wave) was used.

\section{Conclusion}

Using the original method proposed by the Authors of this work, the radius of the electron in motion may be determined with great precision as a function of its speed.

It can be seen from the results listed in Table 1 that the electron does not have a constant radius.

The radius of the electron depends primarily on the speed of travel and in the second row to its rest mass.

The proposed relationship 1 can accurately determines the radius $\mathrm{R}$ of the electron in motion, depending on the speed with which moves $\mathrm{v}$ and its rest mass $\mathrm{m}_{0}$. Mechanical moment of inertia of an electron around of one of its axes could be determined by the relationship 2. Expression 4 shows the total kinetic energy of one electron in motion, as a sum of the two components (translational and rotational). Using the theory of Louis de Broglie, which shows the conservation of the pulse, the wavelength particle associated may be calculated with relationship 7. Wave frequency (associated with the electron in motion) can be determined using the relationship 8. Moving electron kinetic energy may be calculated and through the expression 11, by subtracting the total electron rest energy from total electron energy in movement.

\section{Acknowledgement}

This text was acknowledged and appreciated by Assoc. Pro. Taher M. Abu-Lebdeh, North Carolina A and T State Univesity, United States, Samuel P. Kozaitis, Professor and Department Head at Electrical and Computer Engineering, Florida Institute of Technology, United States.

\section{Author's Contributions}

All the authors contributed equally to prepare, develop and carry out this manuscript.

\section{Ethics}

This article is original and contains unpublished material. The corresponding author approved the manuscript and confirms that no ethical issues involved. Authors declare that are not ethical issues and no conflict of interest that may arise after the publication of this manuscript.

\section{References}

Niels Bohr, From Wikipedia, the free encyclopedia. Retrieved from: https://en.wikipedia.org/wiki/Niels_Bohr

Louis de Broglie, Wikipedia. Retrieved from: https://en.wikipedia.org/wiki/Louis_de_Broglie

Bose-Einstein condensate, From Wikipedia, the free encyclopedia. Retrieved from: https://en.wikipedia.org/wiki/Bose $\%$ E2\%80\%93Ein stein_condensate

Cherenkov radiation, From Wikipedia, the free encyclopedia. Retrieved from: https://en.wikipedia.org/wiki/Cherenkov_radiation

Arthur Compton, From Wikipedia, the free encyclopedia. Retrieved from: https://en.wikipedia.org/wiki/Arthur_Compton

Paul Dirac, From Wikipedia, the free encyclopedia. Retrieved from: https://en.wikipedia.org/wiki/Paul_Dirac

Albert Einstein, From Wikipedia, the free encyclopedia. Retrieved from: https://en.wikipedia.org/wiki/Albert_Einstein

Halliday, D. and R. Robert, 1966. Physics, Part II. 1st Edn., John Wiley and Sons, Inc., New York.

Richard Laming, From Wikipedia, the free encyclopedia. Retrieved from: https://en.wikipedia.org/wiki/Richard_Laming

Lorentz transformation, Wikipedia. Retrieved from: https://en.wikipedia.org/wiki/Lorentz_transformation 
Wolfgang Pauli, From Wikipedia, the free encyclopedia. Retrieved from: https://en.wikipedia.org/wiki/Wolfgang_Pauli

Petrescu, F.I. and J.K. Calautit, 2016a. About nano fusion and dynamic fusion. Am. J. Applied Sci., 13: 261-266. DOI: 10.3844/ajassp.2016.261.266

Petrescu, F.I. and J.K. Calautit, 2016b. About the light dimensions. Am. J. Applied Sci., 13: 321-325. DOI: 10.3844/ajassp.2016.321.325

Petrescu, F.I., A. Apicella, R.V. Petrescu, S. Kozaitis and R. Bucinell et al., 2016. Environmental protection through nuclear energy. Am. J. Applied Sci., 13: 941-946.

DOI: 10.3844 /ajassp.2016.941.946

Petrescu, F.I., 2012. Teoria Mecanismelor Color: Curs si Aplicatii. 1st Edn., CreateSpace Publisher, pp: 284.

Max Planck constant, From Wikipedia, the free encyclopedia. Retrieved from: https://en.wikipedia.org/wiki/Planck_constant

Ernest Rutherford, From Wikipedia, the free encyclopedia. Retrieved from: https://en.wikipedia.org/wiki/Ernest_Rutherford

Thomson, J.J., 1897. From Wikipedia, the free encyclopedia. Retrieved from: https://en.wikipedia.org/wiki/J._J._Thomson

\section{Nomenclature}

$\varepsilon_{0}=>$ the permissive constant (the permittivity):

$\varepsilon_{0}=8.85418 E-12\left[\frac{C^{2}}{N \cdot m^{2}}\right]$

$\mathrm{h}=>$ the Planck constant:

$h=6.626 E-34[J \cdot s]$

$\mathrm{q}=>$ electrical elementary load:

$e=-1.6021 E-19[C]$

$\mathrm{c}=$ the light speed in vacuum:

$c=2.997925 E 8\left[\frac{m}{s}\right]$

$m_{0}[\mathrm{~kg}]=>$ the rest mass of one electron

$m_{0}$ electron $=9.11 \mathrm{E}-31[\mathrm{~kg}]$ 